УДК [62-83:621.313.333]:004.45

DOI $10.21661 / \mathrm{r}-464473$

\title{
Н.Г. Романенко
}

\section{ИМИТАЦИОННЫЕ МОДЕЛИ ПРИВОДА С АСИНХРОННЫМ ЭЛЕКТРОДВИГАТЕЛЕМ ДЛЯ СТУДЕНТОВ ЭЛЕКТРОТЕХНИЧЕСКИХ СПЕЦИАЛЬНОСТЕЙ}

Аннотация: использование виртуальных лабораторий позволит в наглядном виде продемонстрировать различные переходные прочессы, а также провести опыты, которые крайне затратны в реальных лабораториях электрических машин, например, провести расчет энергетических затрат различных типов электроприводов. Рассмотрены модели неуправляемого асинхронного привода, а также частотно-регулируемый асинхронный электропривод. Проведены расчеты и сравнение энергетических затрат этих систем при различных типах нагрузок, что позволяет оценить работу многих технологических механизмов.

Ключевые слова: асинхронный электропривод, частотное регулирование, векторное управление, математическая модель, имитационная модель, Simulink, энергетические потери.

N.G. Romanenko

SIMULATION MODELS OF A DRIVE WITH AN ASYNCHRONOUS ELECTRIC ENGINE FOR STUDENTS OF ELECTRICAL ENGINEERING FACULTIES

Abstract: the application of virtual laboratories will allow to show different transition processes, as well as to carry out experiments that are very expensive in real electrical machines' labs, for example, to calculate the energy costs in electric drives. Models of non-regulation asynchronous drive and frequency-regulated asynchronous electric drive are examined in this article. The author has calculated and compared the energy losses of these systems with various types of loads and this lets us to evaluate work processes of many technical devices. 
Keywords: asynchronous electric drive, frequency regulation, vector control, mathematical system, simulation model, Simulink, energy losses.

На сегодняшний день подготовка электротехнических специалистов невозможна без применения новых форм обучения с использованием компьютерных технологий, базирующихся на современных прикладных программных продукTax.

Современные компьютерные технологии позволяют качественно изменить и существенно улучшить технологию изучения электрических машин, перевести её в виртуальную действительность, осуществить в этой виртуальной лаборатории исследования статических и динамических режимов работы электрических машин, их механических характеристик, условий пуска и технико-экономических показателей с получением количественных результатов.

Использование компьютерных технологий позволит расширить круг и глубину изучаемых вопросов, провести множество экспериментов с использованием виртуальных электрических машин, что благотворно скажется на уровне подготовки специалистов.

В данной статье показан пример виртуальных лабораторных работ для изучения асинхронных двигателей, исследования переходных процессов, естественных и искусственных механических, а также рабочие характеристик. Поставленная задача реализована в средстве визуального программирования моделей - пакете Simulink программы MATLAB.

Рассматриваемые имитационные модели обладают широчайшим спектром возможностей по исследованию асинхронной машины в различных режимах работы, что в реальной лаборатории требует больших финансовых расходов из-за дороговизны необходимого оборудования. Большим плюсом разрабатываемых лабораторных работ является то, что виртуальную лабораторию можно использовать в дистанционном обучении студентов и в различных учебных заведениях.

В зависимости от рассматриваемого механизма, электропривод может работать со следующими типами нагрузок: грузовая, вентиляторная и переменная нагрузка, которую можно описать математической функцией. 
В качестве примера на первом этапе исследуется модель нерегулируемого электропривода работающего с вентиляторной нагрузкой. В качестве приводного двигателя выбран MTKF 512, параметры которого рассчитываются по специальной методике [2, с. 282], а окно настройки показано на рисунке1.

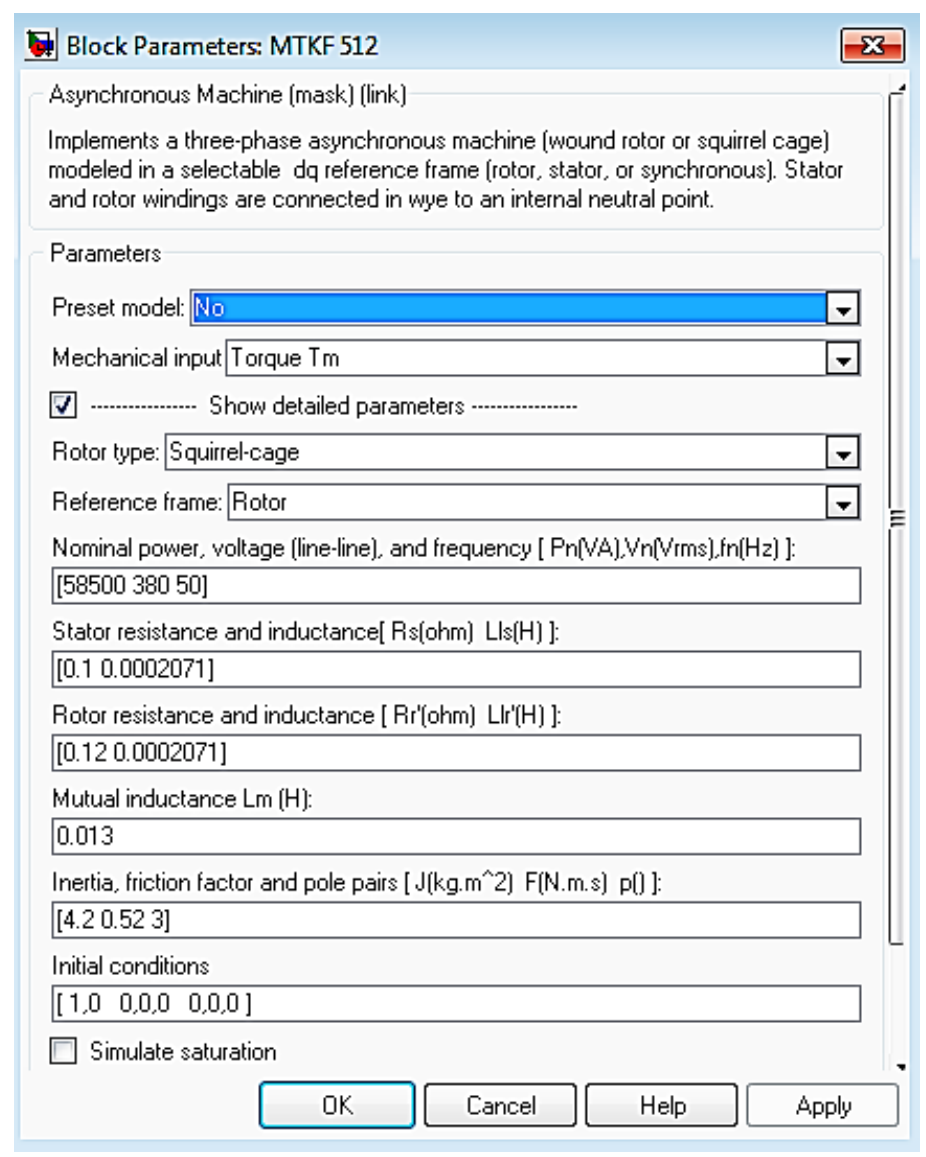

Рис. 1. Окно настройки модели асинхронного двигателя

Модель электропривода представляет собой трехфазный источник электрической энергии, двигатель, блок нагрузки (в данной случае Fcn Function), и осциллографы для получения динамических характеристик (рисунок 2).

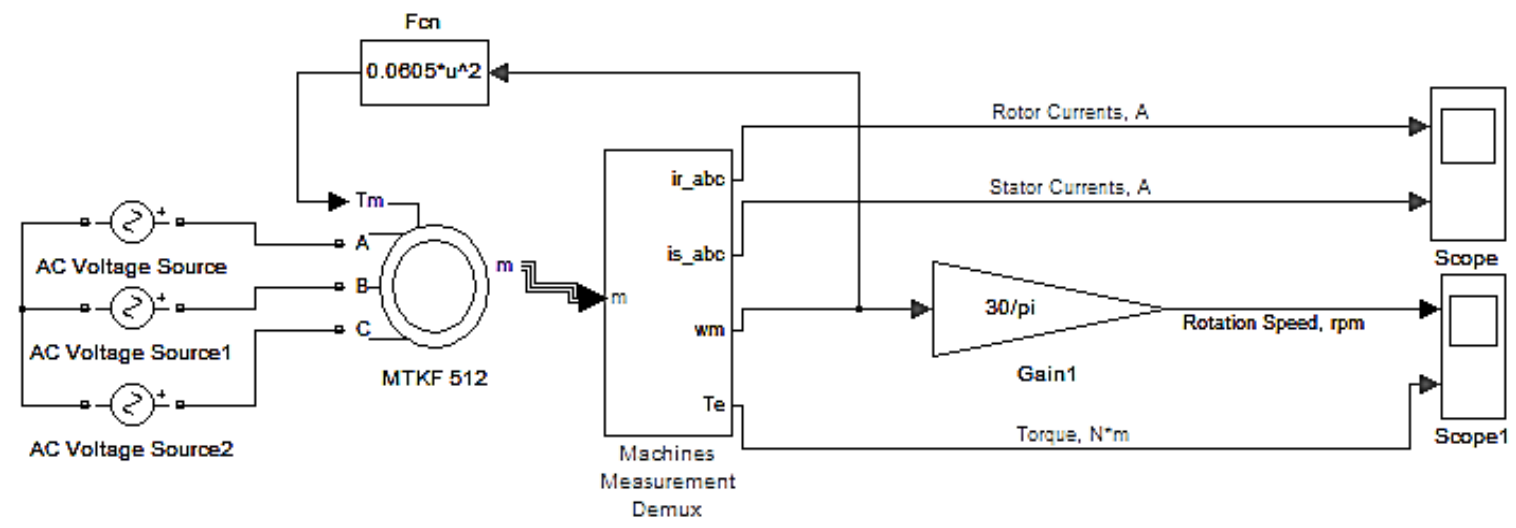

Рис. 2. Модель нерегулируемого асинхронного электропривода 
Учитывая повышенный интерес к проектам по энергоэффективности предприятий актуально применение подобных моделей для расчета электрических затрат и потерь при работе того или иного оборудования. Модель позволяет определить затраты при динамических режимах: пуск, торможение, переход с одной скорости на другую (рисунок 3). Расчет ведется в функции времени, т.е. расчет электрической энергии в $\mathrm{BT}^{*} \mathrm{c}$.

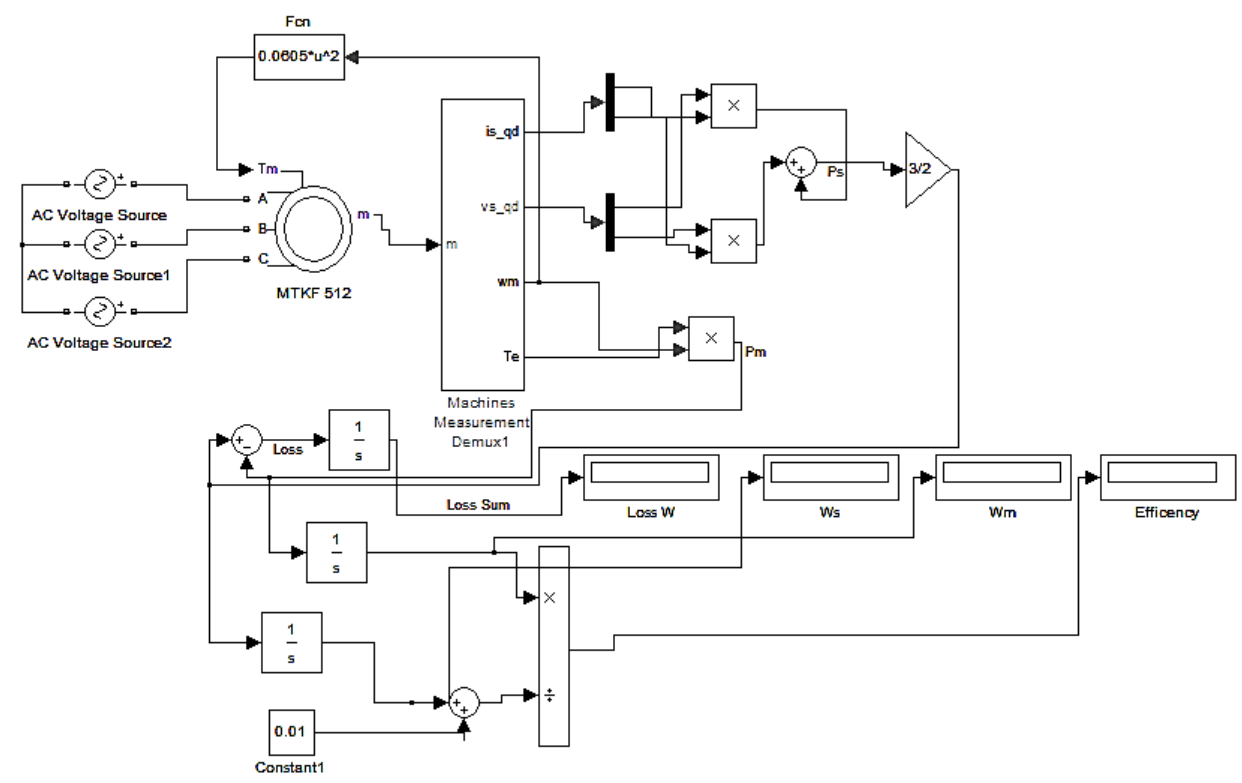

Рис. 3. Модель для расчета энергетических потерь асинхронного электропривода

В результате моделирования получаем параметры Ws - электрическая энергия потребляемая электроприводов из сети, Wm - электромеханическая энергия, efficiency - КПД за цикл работы, Loss W - потери энергии в роторе и статоре. На основании проведенных расчетов полученные данные для одного режима работы представлены в таблице 1. Для наглядности представления результатов измерений построена гистограмма (рисунок 4).

Таблица 1

Энергетические потери нерегулируемого асинхронного привода в функции времени

\begin{tabular}{|c|c|c|c|}
\hline Параметр\Время, c & 1 & 2 & 4 \\
\hline Ws, BT*c & 129219 & 199892 & 341236 \\
\hline
\end{tabular}




\begin{tabular}{|l|c|c|c|}
\hline Loss $\mathrm{W}, \mathrm{BT}^{*} \mathrm{c}$ & 56796 & 65345 & 82470 \\
\hline $\mathrm{Wm}, \mathrm{BT}^{*} \mathrm{c}$ & 72423 & 134567 & 258765 \\
\hline Efficiency, \% & 56,05 & 67,3 & 75,83 \\
\hline
\end{tabular}

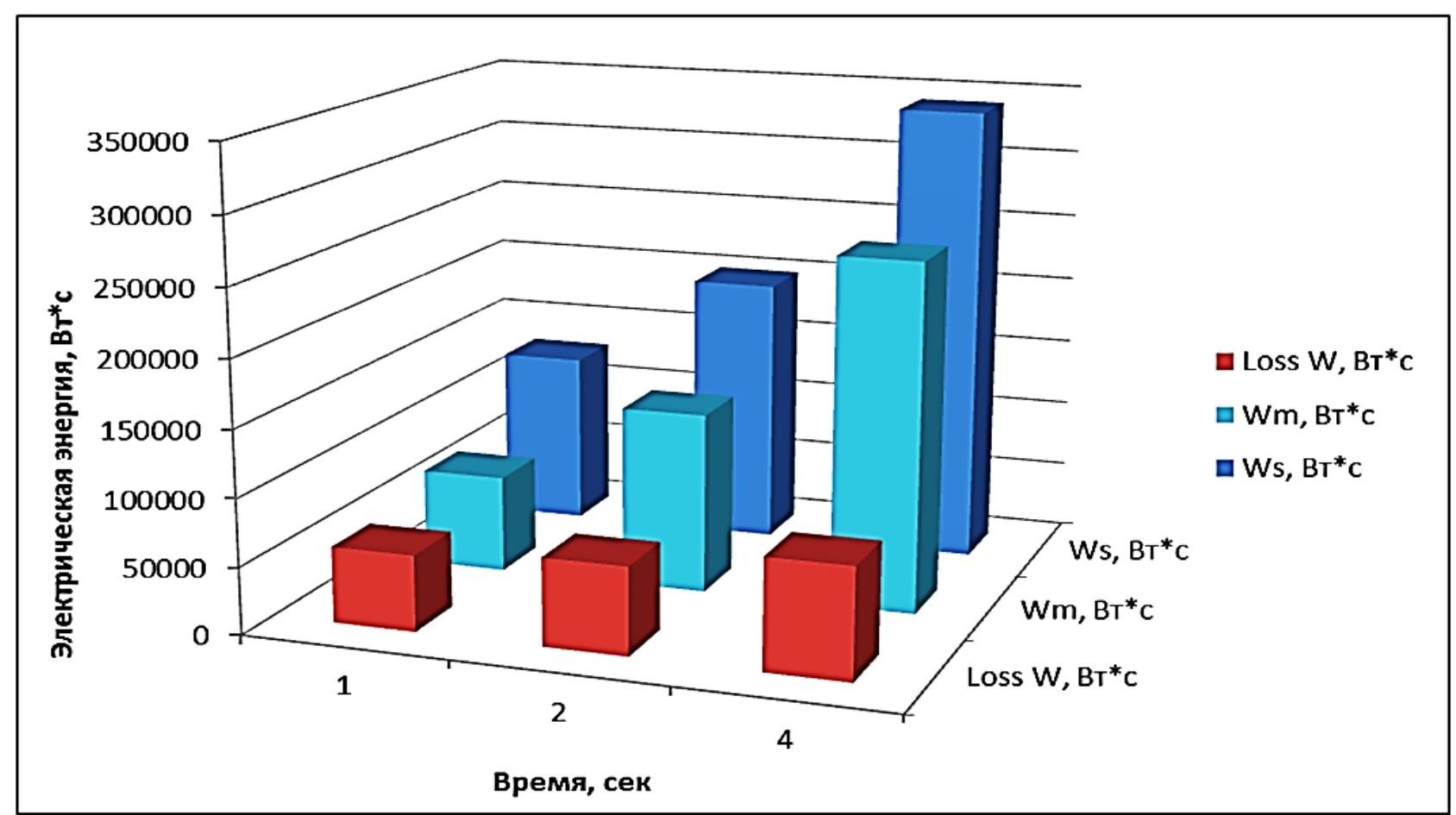

Рис. 4. Гистограмма распределения энергетических потерь от времени нерегулируемого асинхронного привода

Расчет работы неуправляемого электропривода, является основой для сравнения с системой преобразователь частоты - асинхронный двигатель, которая изучаются студентами электротехнических специальностей. Учитывая, что сами преобразователя зачастую довольно дороги для использования их в большом количестве при обучении студентов, применение имитационных моделей позволит сократить затраты и обеспечить необходимым материалом любое количество студентов.

Рассмотрим модель системы асинхронный двигатель - преобразователем частоты для режима векторного управления [1, с. 338]. Модель, обеспечивающая подобный режим работы представлен на рисунке 5. 


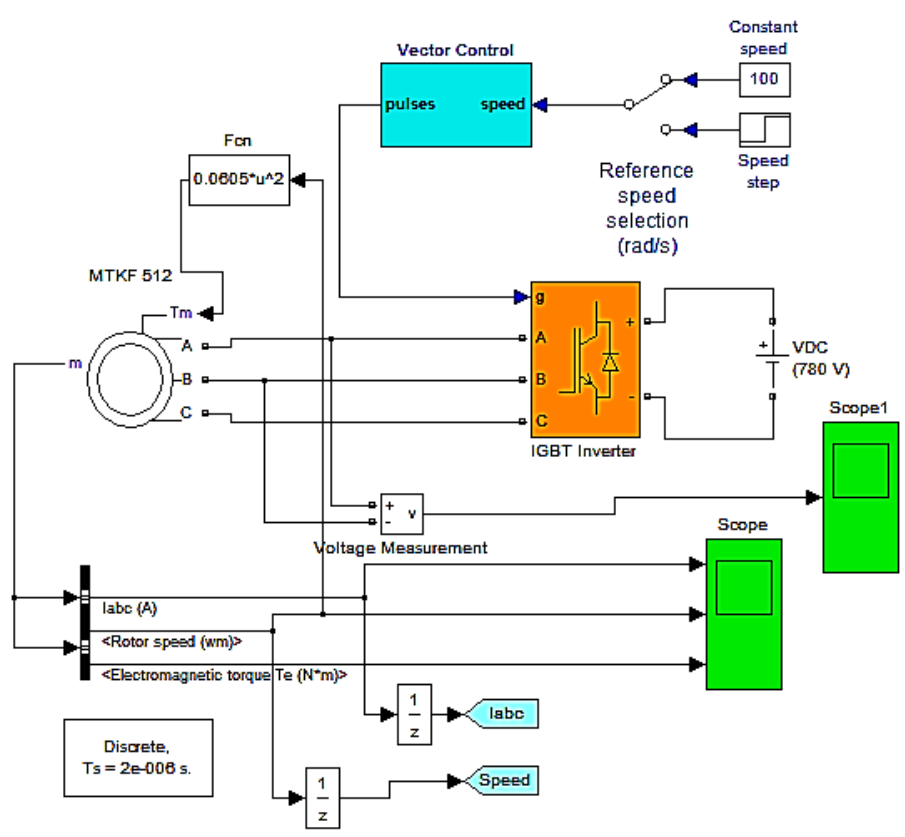

Рис. 5. Модель электропривода при векторном управлении

Основу принципа векторного управления в модели составляет подсистема Vector Control (рисунок 6), в которой используются блоки:

1. Гистерезисный трехфазный регулятор тока (Current Regulator).

2. Блоки преобразования системы координат (ABC to dq conversion, dq to $\mathrm{ABC}$ conversion).

3. Блок определения выходной частоты инвертора (Teta Calculation).

4. Блок вычисления потока (Flux Calculator).

5. Блок задания потока (Phir).

6. Блок регулятора скорости (Speed Contoller).

В блоке Speed Controller используется ПИД-регулятор, настройки которого наиболее сложны при работе с преобразователем частоты. При использовании имитационной модели студенты узнают, изменение каких настроек влияет на характеристики электропривода. 


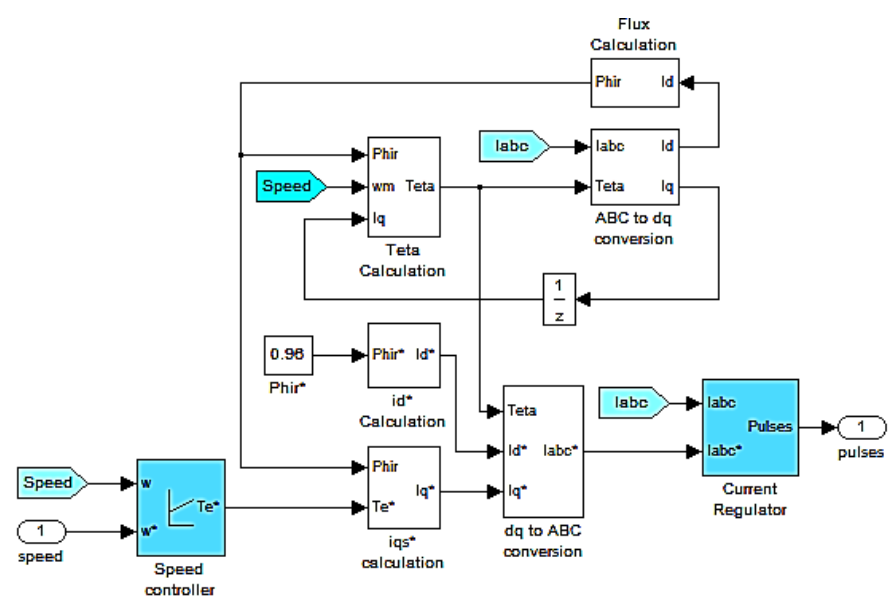

Рис. 6. Внешний вид подсистемы Vector Control

На основании проведенных расчетов полученные данные представлены в таблице 2, для наглядности построена гистограмма (рисунок 7).

Таблица 2

Энергетические потери частотно-регулируемого асинхронного привода в функции времени

\begin{tabular}{|l|c|c|c|}
\hline \multicolumn{1}{|c|}{ Параметр\Время, c } & 1 & 2 & 4 \\
\hline $\mathrm{Ws}, \mathrm{BT}^{*} \mathrm{c}$ & 25157 & 81476 & 241806 \\
\hline Loss $\mathrm{W}, \mathrm{BT}^{*} \mathrm{c}$ & 7083 & 13779 & 31667 \\
\hline $\mathrm{Wm}, \mathrm{BT}^{*} \mathrm{c}$ & 18073 & 67696 & 210148 \\
\hline Efficiency, \% & 72 & 83 & 87 \\
\hline
\end{tabular}

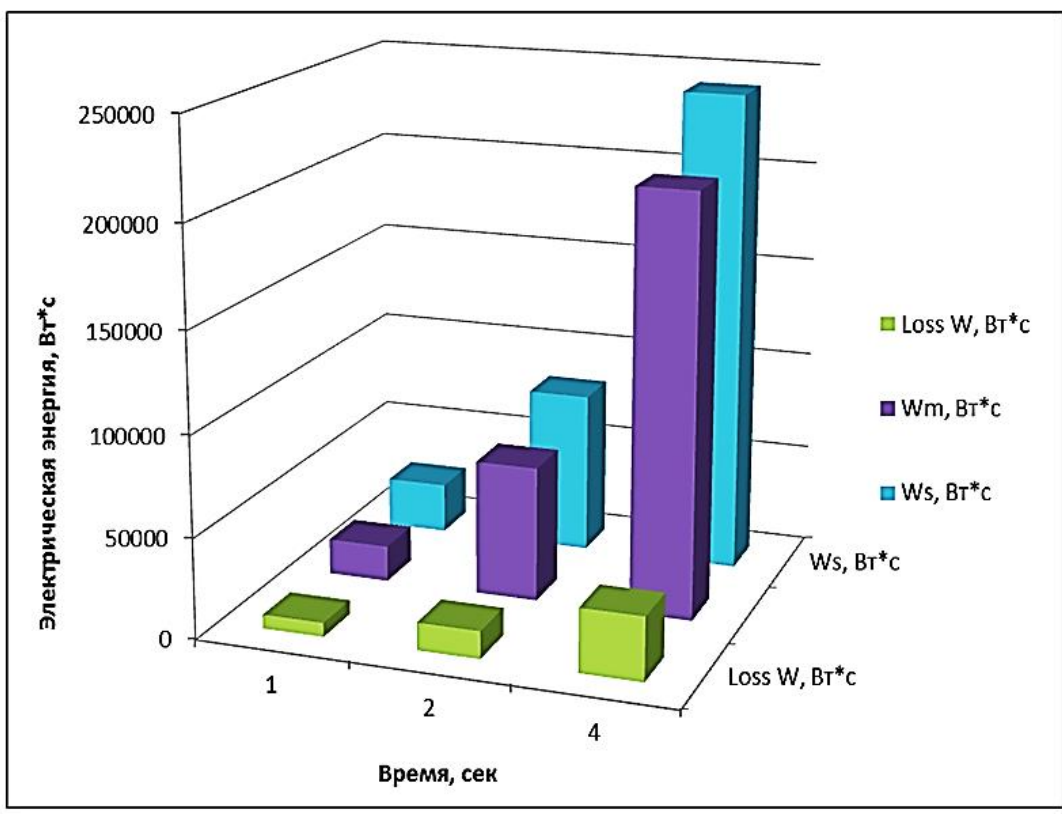

Рис. 7. Гистограмма энергетических потерь частотно-регулируемого электропривода 
Далее необходимо провести сравнительный анализ энергопотребления неуправляемых и частотно-регулируемых асинхронных электроприводов. Подобное сравнение позволяет наглядно продемонстрировать выгоду от использования регулируемого электропривода, а также продемонстрировать возможности использования имитационных моделей для подобных целей. Сравнение для электроприводов, работающих с вентиляторной нагрузкой показано на рисунок 8 .

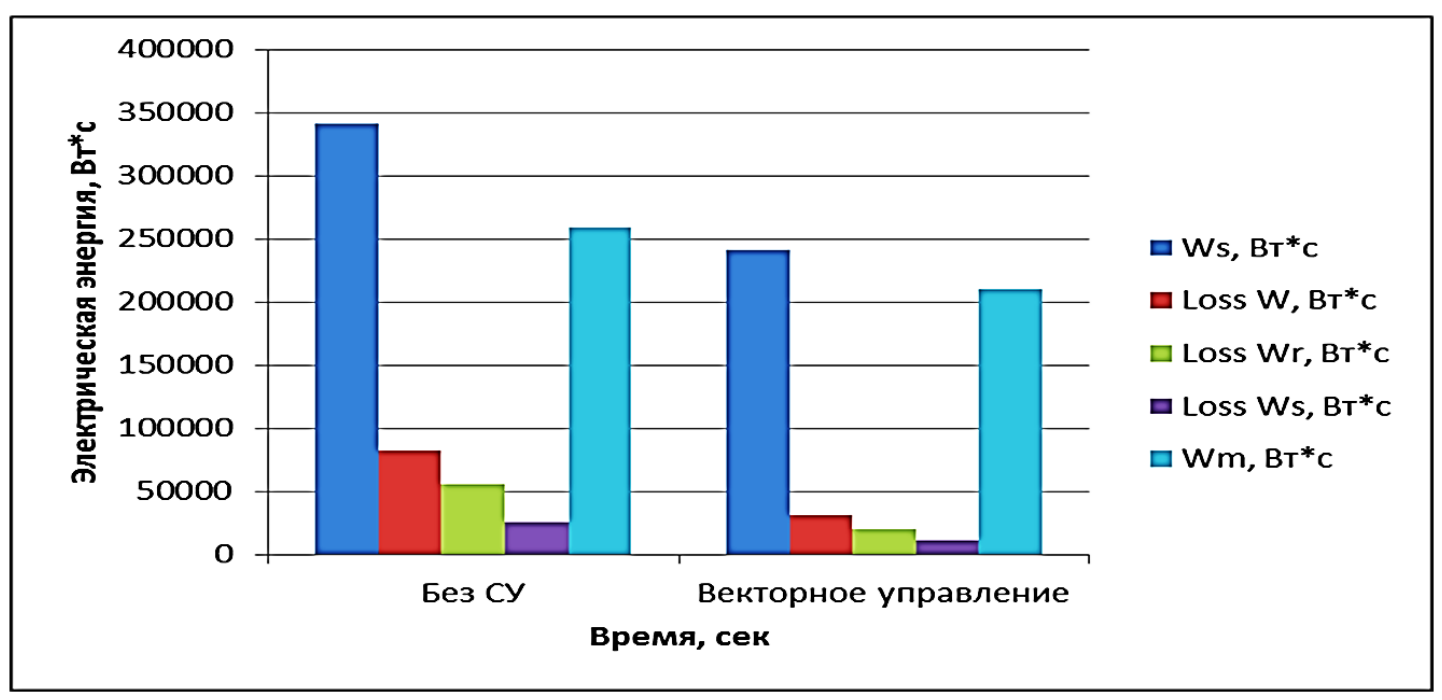

Рис. 8. Сравнение энергетических потерь неуправляемого и частотно-регулируемого электропривода

В заключение следует отметить, что использование имитационных моделей не ограничивается рассмотрением динамических режимов электроприводов в различных режимах работы. Подобные модели позволяют рассмотреть и показать более сложные аспекты работы технологических механизмов [3, с. 17]. Также учитывая возможность непосредственного участия каждого студента в изучении асинхронных электроприводов посредством имитационных моделей, повышается усвояемость теоретического материала, а также улучшаются навыки по настройке и анализу работы электромеханических систем.

\section{Сиисок литературы}

1. Анучин А.С. Системы управления электроприводов: Учебник для вузов. - М.: Издательский дом МЭИ, 2015. - 373 с. 
2. Герман - Галкин С.Г. Matlab\&Simulink. Проектирование мехатронных систем на ПК. - СПб.: Корона-Век, 2008. - 368 с.

3. Надеев А.И. Модернизация электроприводов плавучего крана «Богатырь» с использованием современных энергосберегающих технологий / А.И. Надеев, Н.Г. Романенко, Н.А. Медников, Е.В. Стуров // Вестник Астраханского государственного технического университета. Серия: Морская техника и технология. - 2011. - №3. - С. 16-21.

Романенко Николай Геннадьевич - канд. техн. наук, доцент ФГБОУ ВО «Астраханский государственный технический университет», Россия, Астрахань.

Romanenko Nikolay Gennadyevich - candidate of technical sciences, associate professor at FSBEI of HE “Astrakhan State Technical University”, Russia, Astrakhan. 